INGURUAK [71] | 2021 | 79-100

http://dx.doi.org/10.18543/inguruak-71-2021-art05

ISSN 0214-7912

\title{
Big Data: la revolución de los datos masivos en la Administración Pública
}

\author{
Big Data: The Big Data revolution in Public Administration \\ Lander Jauregi-Maza* \\ Universidad del País Vasco / Euskal Herriko Unibertsitatea (UPV/EHU)
}

\begin{abstract}
RESUMEN: Los datos han sido, son y serán el recurso más importante de una organización, ya sea privada, gubernamental o de cualquier sector. En este trabajo podemos llegar a ver que el Big Data no únicamente nos dirige a los datos, sino que también podemos llegar a encontrar diferentes tecnologías, técnicas, paradigmas y métodos que nos pueden ayudar a resolver diferentes problemas. Pero, para poder hacer uso de estos paradigmas se han llegado a crear diferentes arquitecturas de referencia para poder facilitar el uso del Big Data. Se puede llegar a ver con el paso del tiempo que este fenómeno está adquiriendo mucho peso en el sector público, e incluso de una manera muy acelerada. En uno de los últimos apartados vamos a poder analizar diferentes sectores (turismo, sanidad y gestión urbana) donde el Big Data ha cogido un peso significativo en diferentes países, y cómo ha ido desarrollando su poder. Se puede observar, como nos indicaban los autores MayerSchönberger y Cukier, que esta es la «revolución de los datos masivos».
\end{abstract}

Palabras clave: Big Data, datos abiertos, información, sector público, TIC.

ABSTRACT: Data has been, is and will be the most important resource of an organization, whether private, governmental or in any sector. In this work we can see that Big Data not only directs us to the data, but we can also find different technologies, techniques, paradigms and methods that can help us to solve different problems. But, in order to make use of these paradigms, different reference architectures have been created to facilitate the use of Big Data. It can be seen with the passage of time that this phenomenon is gaining a lot of weight in the public sector, and even in a very accelerated way. In one of the last sections, we will be able to analyze different sectors (tourism, health and urban management) where Big Data has gained significant weight in different countries, and how it has been developing its power. It can be observed, as indicated by the authors Mayer-Schönberger and Cukier, that this is the "massive data revolution».

Keywords: Big Data, open data, information, public sector, ICT.

* Correspondencia a/Correspondence to: Lander Jauregi-Maza. Universidad del País Vasco / Euskal Herriko Unibertsitatea (UPV/EHU) - lander.jauregim@ gmail.com - https://orcid.org/0000-0002-2974-8157

Cómo citar/How to cite: Jauregi-Maza, Lander (2021). "Big Data: la revolución de los datos masivos en la Administración Pública»; Inguruak, 71, 79-100. (http://dx.doi.org/10.18543/inguruak-71-2021-art05).

Recibido/Received: 25 junio 2021; Versión final/Final version: 5 diciembre 2021.

ISSN 0214-7912 / (C) 2021 UPV/EHU

(c) (i) Esta obra está bajo una Licencia

Creative Commons Atribución-NoComercial-SinDerivadas 4.0 Internacional 


\section{INTRODUCCIÓN}

El constante avance de las Tecnologías de la Información y la Comunicación (TIC) ha permitido un crecimiento exponencial en la cantidad de datos generados desde distintas fuentes de información, según la Unión Internacional de Telecomunicaciones, en un análisis ${ }^{1}$ realizado en el año 2019, desde el año 2005 ha aumentado en 36,8 puntos el uso de Internet. Este aumento de la información facilita la toma de decisiones en las administraciones públicas, para lo que los métodos tradicionales de procesamiento de datos han tenido que evolucionar de una manera acelerada. Esta es la base de esa «revolución de los datos masivos» que es el Big Data (Mayer-Schönberger y Cukier, 2013).

El Big Data no solo nos remite a los datos, sino que también encontramos diferentes técnicas, tecnologías, métodos y paradigmas que nos ayudan a poder resolver problemas que se puedan producir.

En torno a estos nuevos paradigmas se han creado arquitecturas de referencia, patrones de diseño y software y hardware para poder facilitar el uso del Big Data, con el objetivo de generar ventajas competitivas y comprender el mundo de una manera más eficiente y eficaz. Pero la eficacia y la eficiencia no resultan simple ni meramente de los datos que son almacenados y gestionados, sino que estos deben ser analizados con el fin de extraer la información que tienen dentro de ellos y del cruce de datos -algo característico del Big Data-y, de esta manera, poder darles el uso eficiente y eficaz que se desea.

Podemos ver que el Big Data está adquiriendo mucho peso dentro del sector público, y de una manera muy rápida. Si observamos, a medida que ha ido aumentando el uso de Internet, mayor cantidad de datos se han ido generando, y mayores archivos se han ido creando para poder guardarlos.

Siempre debemos tener en cuenta que los datos son el recurso más importante para una organización (privada, gubernamental o de cualquier sector), debido a la ganancia potencial que generan de una manera constante. Todas las organizaciones disponen de datos dentro de ellas, y no deben ser descuidados, ya que son uno de los bienes más importantes. Aunque hasta ahora las organizaciones no se hayan dado cuenta que los obtenían de la misma manera que los pueden obtener hoy en día. Aún más, si se parte de la premisa que indica que los datos no siempre generan el valor que se espera de ellos, las organizaciones deben tener la capacidad de descubrirlo en tiempo real sin descuidar aspectos de seguridad, integración, funcionalidad, etc.

Como ejemplos de los beneficios que proporciona el Big Data debe extraerse el valor que los datos generan. En el sector público se han llegado a descubrir diferentes patrones de

${ }_{1}^{1}$ Internet usage keeps growing, but barriers lie ahead. https://itu.foleon.com/itu/measuring-digital-development/ internet-use/ 
carácter demográfico a partir de las redes sociales con los cuales se van a poder analizar de mejor manera a las personas que emiten dichos datos, y con ellos poder generar mucha más información. Asimismo, diarios electrónicos, e incluso, campañas que censan datos para la toma de decisiones de distintos campos. Es decir, para poder generar políticas públicas que se acerquen mucho más a la ciudadanía, ya que con la emisión de sus datos se va a poder tener más información y poder generar de mejor manera las políticas públicas.

El objetivo principal de este artículo es reflexionar sobre el potencial que la implantación del Big Data ofrece a las administraciones públicas. La innovación digital en las administraciones públicas está empezando a ser una realidad, aunque aún se muestre de una manera tímida.

Adicionalmente, también se van a analizar los diferentes usos que se le pueden dar al análisis del Big Data, junto con diferentes ejemplos que hemos podido observar y analizar de forma generalizada en las instituciones públicas mundiales, teniendo en cuenta que las administraciones públicas de todo el mundo han añadido políticas innovadoras relacionadas con el Big Data.

\section{OBJETIVOS, HIPÓTESIS Y METODOLOGÍA}

La innovación en las administraciones públicas está empezando a ser una realidad, aunque aún se muestre de una manera tímida. El Big Data es una de las herramientas innovadoras que podemos observar en los últimos tiempos.

Sin embargo, para poder implantar el Big Data en las administraciones públicas se debe tener en cuenta que los datos son un nutriente muy importante, ya que sin los datos el Big Data no podría llegar a implantarse en ningún sector, ni público ni privado. Esto ya lo estamos viendo hoy en día en nuestra rutina diaria. En todos los trámites que realizamos con la administración estamos ofreciendo datos para que estas instituciones puedan gestionarlos, y de esta manera, poder generar información.

El objetivo principal de este artículo es saber cuál es la presencia y la utilización del Big Data en las administraciones públicas de nuestro entorno y saber cuáles serían las potencialidades que las mismas podrían proporcionar en un futuro cercano y lejano.

Junto con ello disponemos de una serie de objetivos específicos. El primero de ellos es explorar qué penetración (presencia) del uso del Big Data se da en las administraciones públicas. El segundo, saber qué uso se le da al Big Data y cuáles pueden ser sus utilidades. El tercero, poder explorar la evolución histórica del uso de datos en las administraciones. Y finalmente, poder indagar acerca de la implementación de planes para la reutilización de datos. 
Las preguntas de investigación que se plantean son las siguientes:

¿Cómo ha sido la evolución del Big Data hasta nuestros días? ¿Cuál es el verdadero significado del Big Data? ¿Cuál es la relación que tiene el Big Data con las administraciones públicas? ¿Es capaz el Big Data de generar cambios significativos en el sector público?

Siguiendo el hilo argumental, y en busca de soluciones para algunas de estas incógnitas, en este artículo haremos uso de las siguientes hipótesis:

- Hipótesis 1: a mayor extensión de la tecnología, mayores posibilidades de extensión habrá de las herramientas del Big Data de manera global.

- Hipótesis 2: dado que los meros datos no llegan a proporcionar conocimiento, a medida que necesitamos mayor y más preciso conocimiento técnico y especializado, más necesidad tenemos del uso de las herramientas que proporcionan Big Data.

- Hipótesis 3: la aplicación del Big Data en diferentes sectores de servicios públicos está ofreciendo resultados notables en la proporción de servicios públicos y de bienestar general.

— Hipótesis 4: el Big Data no da los mismos resultados en todos los sectores. Se considera más adecuado para determinados sectores.

La metodología de este estudio será de carácter estructural, lo que nos va a permitir tomar en consideración aspectos que no siempre son de carácter cuantificable, como los políticos, los sociales, los institucionales, los geográficos, los históricos, etc. Esto nos permitirá saber cuál ha sido el recorrido del Big Data hasta nuestros tiempos, y saber qué tipo de impactos ha tenido en las administraciones públicas. Para ello, haremos uso de diferentes técnicas cualitativas de recogida de datos, y de esta manera, poder observar y responder a los diferentes objetivos, preguntas de investigación e hipótesis planteadas en nuestro trabajo.

La variable "uso del Big Data» que está inmersa en todas las hipótesis, será la variable dependiente del estudio, que podremos ver cómo evoluciona haciendo uso de diferentes variables independientes (administraciones públicas, tiempo, uso de las TIC, etc.). De esta manera, podremos conocer qué evolución ha tenido el uso del Big Data en el sector público, así como los beneficios para poder realizar mejores políticas para la ciudadanía.

\section{CUANDO HABLAMOS DE BIG DATA, ¿A QUÉ NOS REFERIMOS?}

Se conoce con este anglicismo el conjunto de datos cuyo tamaño llega a superar la capacidad de almacenamiento, búsqueda, gestión, análisis, captura o, incluso, la protección legal de 
las herramientas convencionales de gestión de cualquier tipo de archivo (Bauzá, 2017: 21). Cuando hablamos de Big Data nos referimos a las características principales que disponen los datos con los que trabajamos, haciendo especial referencia a sus características definitorias: las siete uves (volumen, variedad, velocidad, visualización, variabilidad, valor y veracidad). Pero, en la mayoría de ocasiones nos olvidamos de atender a la materia prima con la que tenemos que trabajar, es decir, los datos.

Si comenzamos haciendo referencia a lo que pensamos que es un dato, podríamos partir de su acepción etimológica del latín datum, que significa «lo que se da». Pero hacer uso de un término tan lejano para hacer referencia a un concepto tan novedoso como es el Big Data puede ser un poco inapropiado. Por lo tanto, para continuar con nuestro análisis, vamos a hacer referencia a las definiciones que nos aporta la Real Academia de la Lengua Española (RAE) sobre el dato, donde se define como (1) «información sobre algo concreto que permite su conocimiento exacto o sirve para deducir las consecuencias derivadas de un hecho», (2) «documento, testimonio, fundamento» y (3) «información dispuesta de manera adecuada para su tratamiento por una computadora». De manera más clara, podemos ver que la tercera definición que nos da la RAE es la que más la podemos asociar con el Big Data, por el hecho de que nos indica que los datos van a ser analizados por una computadora. Pero, el resto de las definiciones también nos indican cosas relevantes, tales como que los datos pueden llegar a ser documentos o testimonios, además de que en dos de las tres acepciones se hace uso de la palabra «información».

Sin embargo, es muy importante diferenciar entre dato e información. Russell L. Ackoff, académico estadounidense, introduce tres términos que de una manera informal se llegan a tratar de una manera diferente, pero que en un ámbito más formal son distinguibles: datos, información y conocimiento. ${ }^{2}$

Gráfico 1. La pirámide del conocimiento

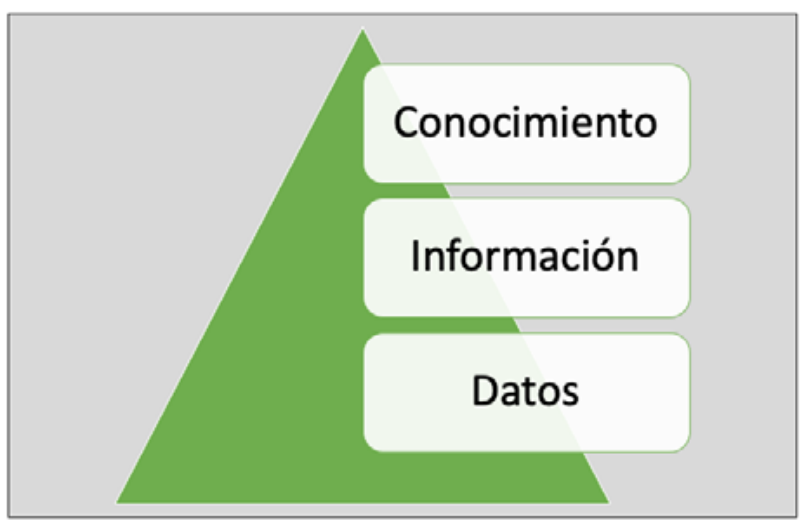

Fuente: elaboración propia, a partir de Ackoff (1989).

\footnotetext{
2 Ackoff presentó en la pirámide del conocimiento cuatro conceptos: datos, información, conocimiento y sabiduría. Pero, en este trabajo únicamente se hace uso de los tres primeros conceptos. El por qué de esta decisión se debe a la confusión que genera el conocimiento y la sabiduría en lo alto de la pirámide.
} 
En el Big Data hacemos uso de todo tipo de datos que proceden de archivos de administraciones públicas y de organismos privados, y que, en ocasiones, dichos datos, pueden estar en portales de Open Data o Transparencia disponibles de distintas maneras en las páginas web en modo descarga: en formatos estructurados o entrelazados, con textos de las páginas digitales, etc.

Los datos los podemos disponer de diferentes maneras en las páginas web de Open Data de las diferentes Administraciones Públicas. Si nos sumergimos en alguna de ellas podemos encontrar diferentes temáticas, de las cuales podemos extraer diferentes archivos para poder generar información. Los diferentes formatos (XML, CSV o JSON) que queramos descargar van a depender del programa informático con el que queramos trabajar. Estos podrían considerarse elementos primarios con los que vamos a trabajar en la normalidad. De hecho, ocasionalmente se suele hablar de la distinción de raw data (datos en crudo) y datos que ya han sido trabajados, refiriéndonos a los datos que ya han sido limpiados, agregados, etc.

Pero dado que los datos por si solos no nos proporcionan mero conocimiento, a medida que necesitamos mayor, y más preciso conocimiento técnico y especializado, tenemos más necesidad del uso de las herramientas que proporcionan Big Data.

Los datos no tienen mucho valor si no se les puede generar un significado. De nada sirve que tengamos atributos y sus valores, si no se conoce qué es lo que nos quieren decir y cómo se puede llegar a interpretar. Por ejemplo, el nombre de una persona o el numero de teléfono de ella sin un propósito, una utilidad o un contexto no sirven de nada para apoyar la toma de una decisión. Los datos, como se ha mencionado, pueden ser una colección de hechos que han sido almacenados en un lugar físico (como un papel), un dispositivo electrónico (archivo online, pendrive, disco duro...), o incluso, la mente de una persona. En este sentido las TIC han aportado mucha recopilación de datos.

Si continuamos ascendiendo en la pirámide, la información está «contenida en descripciones, dando respuesta a preguntas que comienzan con palabras tales como quién, qué, dónde, cuándo, cómo y cuántos» (Ackoff, 1989: 3). El contexto puede llegar a variar desde la descripción contextual de los diferentes encabezados de las columnas de un archivo donde se muestran los datos, e incluso, desde los atributos, hasta las descripciones que están tipificadas como más detalladas de la metodología que se ha llegado a seguir para poder conseguir el conjunto de datos, la descripción de los valores, etc. También podríamos considerar información cualquier tipo de datos que podamos extraer de datos simples (noticas de prensa, informes de las administraciones, etc.).

El siguiente punto por analizar son los conocimientos. Según Ackoff, conocimiento es «lo que hace posible la transformación de la información en instrucciones» (Ackoff, 1989: 4). Además, por este término podemos entender la fusión de la información y el know-how (saber cómo) que nos permita entender de mejor manera los datos y la información que tenemos, y de esa manera poder aplicarlos a nuestra institución (Ackoff, 1989). 
Cuando nos referimos a Big Data, parece que siempre nos referimos a la disponibilidad de los datos, y según los cuales se pueden hacer todo tipo de análisis que sean necesarios para poder obtener información, y con dicha información, generar conocimientos. En casi todos los países del mundo ha surgido la iniciativa con los datos abiertos, en la mayoría de ellos liderados estos proyectos por las administraciones públicas, que han generado normativas para poder hacer públicos los datos que disponen mediante licencias abiertas, y de esta manera, poder reutilizar los datos, la rendición de cuentas y la generación del valor económico y social (Mirón, 2017).

Aunque debemos tener en cuenta que sin los datos poco podríamos llegar a hacer, ya que se están generando de una manera constante y en cantidades muy grandes, por ello el valor de las técnicas de recogida del Big Data están aumentando de manera persistente. Podríamos decir que disponemos de una relación directa entre las técnicas del Big Data y los datos. A medida que los datos van en aumento, las técnicas de Big Data van en aumento. Pero tampoco debemos olvidar el contexto que rodea todos esos datos; es decir, lo que los convierte en información, además también disponemos de los conocimientos que se pueden llegar a derivar en diferentes organizaciones, ya sean de ámbito público o privado, y puedan convertirse disponibles para que otros la utilicen.

Gráfico 2. Aplicar la pirámide del conocimiento a la realidad de la pobreza

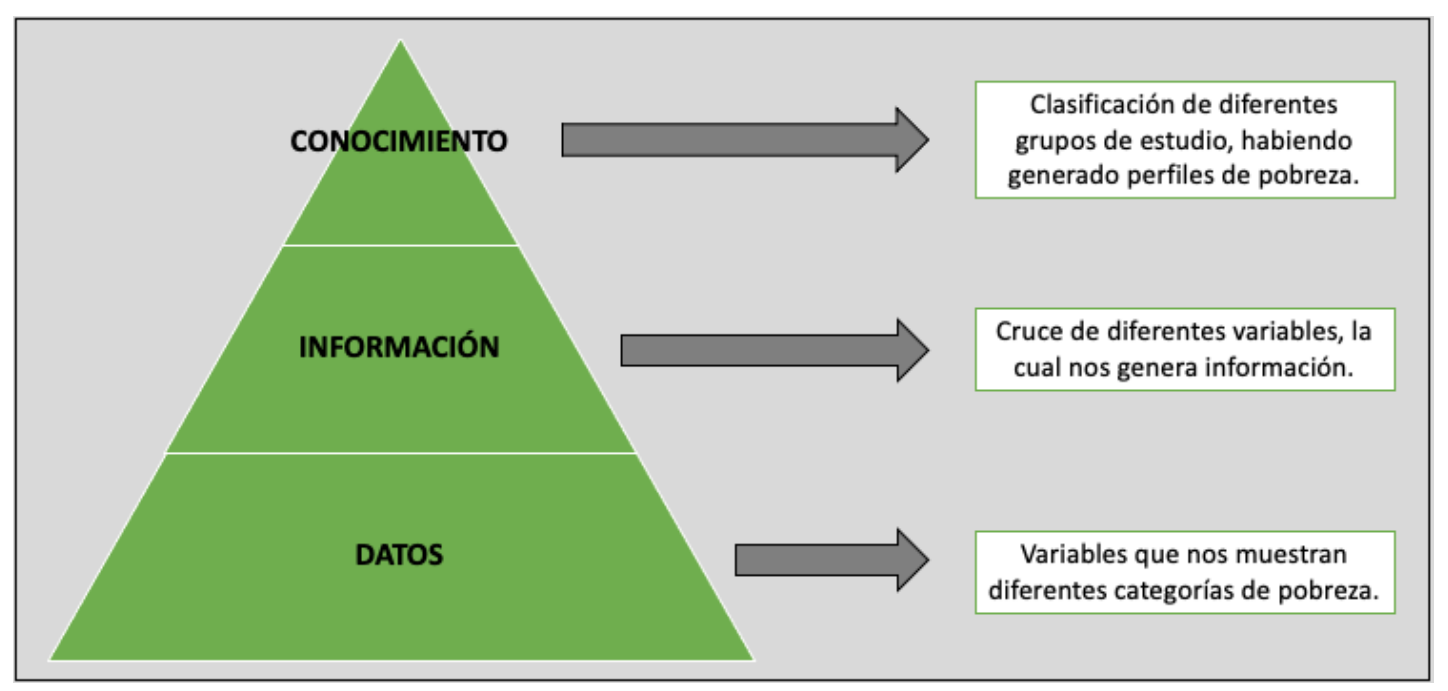

Fuente: elaboración propia.

Si observamos diferentes ejemplos de los tres puntos mencionados de «La pirámide del conocimiento» de Ackoff (datos, información y conocimiento), podemos ver cómo una temática puede llegar a evolucionar y poder diferenciarse detenidamente en cada uno de ellos. Aquí podemos ver que las políticas públicas de pobreza las podemos visualizar en datos, información y conocimiento.

-Datos: variables que nos muestran únicamente diferentes categorías de pobreza. Ejemplo: nivel de pobreza en Euskadi, edad de las personas en Euskadi, número de personas residentes en casas, etc. 
-Información: cruce de diferentes variables, las cuales nos llegan a mostrar información. Edad de las personas según su nivel de pobreza. Ese cruce de variables nos llega a mostrar una información muy importante.

- Conocimiento: con la información generada con los datos, podemos clasificar a las personas en diferentes grupos de estudio, habiendo generado diferentes perfiles de pobreza.

\section{EL BIG DATA EN EL SECTOR PÚBLICO}

El Big Data en el sector público no ha llegado a tener gran impacto, pero nuestra sociedad y, sobre todo, los representantes públicos y privados han abordado esta cuestión como una de las principales de nuestros días. En este apartado podremos observar tres temáticas principales del Big Data en el sector público que tienen gran calado para que pueda introducirse de manera eficaz y eficiente. La primera de ellas es su estado en las administraciones públicas en nuestros días. En segundo lugar, para qué puede pueden llegar a ser importantes las infraestructuras y plataformas tecnológicas dentro de la recogida de datos masivos. Y finalmente, cómo debería ser regulado el uso del Big Data en el sector público.

Representantes de sector público y del sector privado han abordado la cuestión desde la consideración del Big Data y la analítica de datos como una de las cinco tecnologías disruptivas (Big Data, Movilidad, Social Business, Cloud e Internet of Things) cuyo impacto en la sociedad se hará notar de una manera especial en los próximos años (OSPI, 2016).

Respecto a la gran cantidad de datos que disponemos hoy en día en diferentes órdenes de la vida, los expertos coinciden en señalar unas características que hacen algo único con un carácter muy particular, siendo la creación de una nueva escala en los datos, es decir, los Terabytes. Disponer de este elemento es, sin ninguna duda, disponer de algo muy grande, tan grande como las dudas que se generan de cómo se deben utilizar, además de cómo se deben gestionar esos datos, quién debe o puede acceder a ellos, cómo legislar la utilización de dichos datos, etc. Sin duda alguna, el Big Data puede proporcionarnos un gran número de beneficios y ayudas a las administraciones públicas y a la ciudadanía. No obstante, junto a esos beneficios, también se suelen crear diferentes interrogantes para poder gestionar esos datos.

De todas maneras, y para poder contextualizar la magnitud que tiene el Big Data, una de las primeras cuestiones que se deben tener en cuenta es que no se debe perder la perspectiva del dato como nutriente y parte fundamental del Big Data. La territorialización del dato es un elemento clave para poder avanzar en los proyectos que se vayan a hacer en esta línea.

Los especialistas en la materia coinciden en resaltar, como lo hace la Organización para la Cooperación y el Desarrollo Económicos (OCDE) en su informe sobre Open Government 
Data: Towards Empirical Analysis of Government Data Iniciatives (Ubaldi, 2013), el valor de la apertura de datos del sector público, definido a través de cinco elementos clave (OSPI, 2016):

- Mejora de responsabilidad de diferentes gobiernos, la transparencia y el control democrático.

—Promoción del empoderamiento y la participación ciudadana.

- Creación de una nueva generación de empleados públicos que sean competentes para esta nueva generación.

—Fomento de la innovación, la eficiencia y la eficacia en los servicios públicos.

- Creación de valor para la totalidad de la economía.

De esta manera, como hemos visto en el anterior apartado del artículo, debemos ser capaces de gestionar y transformar los Terabytes de datos en una información, y después, en conocimiento para poder aplicarlos a diferentes ámbitos de la gestión pública como el tributario, la sanidad, la seguridad, etc. El Big Data pone a disposición de cualquier administración pública la información que se considera de valor para poder llegar a tener mucha más productividad, entendiendo la productividad de la administración pública desde el prisma de la mejora de la calidad en los servicios que presta a la ciudadanía.

Además, uno de los mayores retos ha sido acercar los datos a los procedimientos administrativos y reducir los tiempos de respuesta, llegando a ampliar de manera simultánea la capacidad de juntar todo tipo de información.

En el sector público se llega a avanzar en aspectos que están relacionados con la normalización y la producción estadística, en los que el Big Data tiene cada vez mayor peso (OSPI, 2016). Pero, uno de los frenos a ese avance es la identificación de distintos casos de uso, es decir, saber cómo, cuándo y para qué utilizar esa tecnología. La línea que marca el avance parece que se llega a situar en la combinación entre lo tradicional y lo novedoso, y las fórmulas que lleguen a facilitar la adopción de lo nuevo. Para ello es importante regularizar el uso que se le va a proporcionar al Big Data dentro de la producción estadística, ya que, sin la regularización oportuna, se podrían crear situaciones en las cuales no se podría encontrar una solución para poder corregirlas.

Hoy en día, existen pocas prácticas y gestión de información que nos permitan testar la generación de conocimiento que disponemos a través del Big Data. De todas maneras, es necesario impulsar un cambio que permita pasar de tener mucha información a tener conocimiento de esa información. De esta manera, la tecnología debe representar la información, sintetizarla, agruparla, someterla a criterios de fiabilidad, eliminar la errónea, procesarla y mostrar lo que realmente se considera relevante.

La administración debe disponer de unos estándares de privacidad que se encuentran prefijados, al igual que las leyes de protección de datos. Hoy en día, las fuentes de infor- 
mación son diversas y múltiples, pero también disponen de una capacidad mucho más grande para poder almacenar datos, lo que nos permite, de una manera continua, seguir produciendo y guardando grandes volúmenes, ya sean de fuentes internas o externas. La tecnología la podemos considerar que está madura, pero debemos tener bien claro y definido el objetivo que tiene el Big Data, que bien podría resumirse en la necesidad de indexar diferentes fuentes de información en un tiempo que sea útil. El tiempo útil puede coincidir con el tiempo real, pero no tiene por qué coincidir.

La aplicación del Big Data al sector público debe ser práctico y abordar proyectos en los que realmente se produzca una aportación de valor que sea efectiva. Junto a ello, la tecnología debe permitir procesar los datos en crudo, e incorporarlos a la actividad del análisis. Es decir, los datos en crudo deben convertirse en datos estructurados para poder incorporarlos a las herramientas de análisis de cada administración pública con el objetivo de construir y definir diferentes y novedosos indicadores.

Pero, alcanzar la parte inductiva es otro de los grandes retos que también nos genera el Big Data. Dicho de otra manera, buscar los patrones relevantes. La tendencia actual es guardar toda la información que sea posible, mientras que la tecnología debe contribuir a extraer el valor que disponen los datos. Pero, se genera una pregunta: ¿cómo se puede tratar el análisis de toda esa información? Para poder responder a esta pregunta, se perfila otra necesidad que hay que cubrir de manera imperiosa, y es la carencia de personal cualificado que nos permita dar una respuesta a los requerimientos que plantea el nuevo escenario en el que nos encontramos.

De los nuevos perfiles profesionales destaca la formación como ingeniero de datos y la habilidad para poder indagar en el conocimiento técnico y el conocimiento de negocio. En el entorno privado se empieza a configurar la figura del Chief Data Officer (CDO, en adelante) como la persona que es propietaria de los datos, porque facilitar el acceso a la información dentro de cualquier empresa u organización debe ser primordial. Este rol del CDO también lo podemos ver en la administración pública. En el sector público lo podemos definir como el encargado de generar una visión única para los distintos consumos de datos que se vayan a generar.

Finalmente, para poder alcanzar el éxito de los proyectos de Big Data se debe implicar, no a las administraciones públicas y las empresas privadas, sino también al conjunto de la sociedad, ya que son los consumidores de datos y de información, pero también son generadores de dicha información. Los expertos lo consideran como «inteligencia colectiva», por supuesto, llegando a garantizar la seguridad y racionalizando los diferentes proyectos. La participación colectiva va a llegar a impulsar los nuevos usos que se le pueden dar al Big Data, que evolucionará de los diferentes análisis retrospectivos a los análisis predictivos.

La integración es una parte fundamental para poder continuar con el análisis del Big Data. Es decir, la integración es la capacidad de poder hacer que en una misma base de datos 
pueda combinarse todo tipo de información, siendo de cualquier tipo (operacional, analítica o de riesgo).

Pero, hablar sobre la infraestructura es hablar sobre un ecosistema de tecnologías que ya se encuentran disponibles en el sector público, y que permiten que sea la propia tecnología la que se llegue a adaptar a los casos de uso, llegando a hacer que los proyectos puedan ser llevados a cabo en plazos cada vez más rápidos, o incluso, mantener una constancia en ellos. Antiguamente, una única base de datos relacional servía para solucionar cualquier tipo de problema. Hoy en día, estamos más avanzados tecnológicamente, disponiendo de diferentes bases de datos orientadas a grafos ${ }^{3}$ o basadas en SQL ${ }^{4}$ (Structured Query Language). De esta manera, es importante explotar los datos en el modo low cost. Como podemos observar en el sector público, cada día avanza de una manera mucho más rápida el uso de las TIC y de los datos para poder tener proyectos y políticas públicas mucho más eficaces y eficientes.

Tarde o temprano, las infraestructuras diseñadas para la gestión del Big Data van a acabar por incorporarse a empresas privadas y a la administración pública, incluso algunas ya se han sumergido por completo en estas tecnologías. Estas incorporaciones se van a realizar por razones de coste o por identificación de diferentes casos de uso. Pero, habrá que tener solventadas algunas cuestiones como la convergencia y la manejabilidad, la escalabilidad y la seguridad, etc.

Un aspecto que se considera clave está inmerso en la seguridad y la convergencia hacia un lenguaje conocido y utilizado para acceder a los datos. De manera que, aunque se establezca una nueva infraestructura, siempre se va a tener en cuenta el criterio de la convergencia, para que el sistema pueda ser manejable y útil. Y en este caso, podemos establecer que el SQL es la opción más viable (OSPI, 2016).

Además, se debe tener en cuenta la dificultad que entraña en el hecho de convencer a los responsables de la administración pública que manejan los presupuestos, es decir, de los políticos, sobre la necesidad de la utilización de las tecnologías en la administración pública. Se deberán buscar nuevos argumentos que nos posibiliten mostrar el valor que tiene el Big Data con diferentes evidencias y hechos.

Un aspecto que preocupa de forma general a todas las personas es la seguridad y, sobre todo, la protección de los datos. Cientos de millones de datos están en continuo movimiento, y muchos de ellos se transmiten desde redes de nula seguridad, como pueden

\footnotetext{
3 Según Wikipedia, los grafos son un «conjunto de objetos llamados vértices o nodos unidos por enlaces, llamados aristas o arcos, que permiten representar relaciones binarias entre elementos de un conjunto». Disponible en: https://es.wikipedia.org/wiki/Grafo

${ }^{4}$ Según Wikipedia, la SQL es un «lenguaje de dominio específico utilizado en programación, diseñado para administrar, y recuperar información de sistemas de gestión de bases de datos de carácter relacional». Disponible en: https://es.wikipedia.org/wiki/SQL
} 
ser las Wifi de dominio público. Si bien es cierto, dichas Wifi nos permiten navegar por Internet desde dispositivos móviles, otra realidad por la que también la ciudadanía tiene preocupación por la seguridad inmersa en ella. Pero, no solamente existen estas preocupaciones, sino que el Internet de las Cosas (IoT) es otra cuestión importante dentro de la seguridad y privacidad escasa que pueden tener los hardware (Beltrán-Orenes y MartínezPastor, 2016).

En estos momentos, el poder se llega a concretar en las grandes empresas que son impulsoras de las distintas plataformas tecnológicas que tenemos a nuestra disposición, ya que han mostrado su capacidad para poder recopilar datos, hasta el punto de producirse la paradoja de que este tipo de organizaciones podría disponer de más información que la propia Agencia Tributaria, ya que ésta es una administración que posee el mayor volumen da datos sobre la ciudadanía española, además de las empresas (Cotino, 2019). De manera probable, este desequilibrio nos puede conducir a una revisión del marco normativo. Esta revisión puede estar orientada a la búsqueda de diferentes y novedosas fórmulas de equilibrio entre la información que manejan los sectores públicos y privados.

Pero los escenarios que la ciudadanía demanda con la administración pública obligan a implementar este tipo de prácticas, aún con los riesgos que conllevan. Un ejemplo de esta práctica en el ámbito público puede ser en la sanidad (Groves et al., 2013). La ciudadanía solicita que en el ámbito de la sanidad se haga uso de los teléfonos móviles para una mayor comunicación y de una manera constante. Esto ayuda, por poner un ejemplo, a que la ciudadanía esté en todo momento informada de su situación sanitaria, y pueda consultar en cualquier momento su expediente sanitario.

En cualquier caso, la movilidad es un hecho que no podemos obviar, pero tenemos que tomar medidas para poder minimizar los riesgos, además de gestionarlos de una manera eficiente, junto con guardar los datos con las garantías de seguridad suficientes para poder tenerlos a buen recaudo. Estas medidas deberán ser de distintos tipos: técnicas, legales, organizativas, de formación, etc. No podemos dejarlo todo en manos de la justicia, ya que la ley va a evolucionar, pero el Big Data evoluciona de manera mucho más rápida, por lo tanto, cuando queramos fijarnos en la ley, va a estar anticuada para el momento en el que nos encontremos.

Otra cuestión que no podemos dejar atrás es la idoneidad de compartir los datos. De esta manera, ¿debería la administración pública convertir los datos de ámbito público en un activo para el resto de las personas? Por supuesto, como se ha mencionado en el anterior párrafo, deberían ser totalmente anónimos, para poder salvaguardar la privacidad de las personas. Un informe elaborado por la OECD muestra que existen diferentes evidencias de que con una completa explotación de los datos públicos, las administraciones públicas podrían llegar a reducir de una manera significativa sus gastos de funcionamiento. El informe nos indica que, para los 23 mayores Estados de la Unión Europea, este ahorro puede situarse entre un 10\%-15\% (OECD, 2013: 5). 
En España se comparte más información de la que podría pensarse que aporta la administración pública. Este traspaso de datos se realiza mediante diferentes convenios entre las administraciones públicas. De esta manera se ha realizado y se seguirá realizando un esfuerzo para poder potenciar el intercambio de información entre las administraciones, además del acceso a la información, llegando a minimizar el delito de fraude o falsificación de datos. Una de las infraestructuras que se ha llegado a crear con todo este recorrido es el Portal de Administración Electrónica ${ }^{5}$ del Gobierno de España.

Con referencia a los datos abiertos hay otra cuestión que no podemos dejar en el olvido, que no se considera tanto tecnológica como de negocio y que tiene relación con el uso que la ciudadanía pueda dar a esos datos. La generación de negocio es un importante argumento para poder seguir hacia delante en la apertura de los datos, si bien en esto las administraciones públicas deben actuar con mucha prudencia, ya que ellas son las instituciones que salvaguardan datos que requieren distintos niveles de seguridad.

Finalmente, aunque la norma general recogida en la normativa sobre la reutilización de datos públicos es la de la gratuidad del acceso, se ha comenzado a poner en marcha algunas alternativas de colaboración de ámbito público-privado. Se ha hecho bastante hincapié en organismos a los que no se aplica la norma general. Son alternativas que están permitiendo disminuir el tiempo de los plazos para la puesta a disposición de información y documentación.

\section{EL USO DEL BIG DATA EN EL SECTOR PÚBLICO}

Las instituciones públicas hacen uso de los datos de manera intensiva, y cada vez más instituciones en todos los niveles de gobierno (nacional, autonómico y local) están tratando de aprovechar la información para poder dar un mejor servicio a la ciudadanía. Las acciones se centran en garantizar la seguridad y privacidad de los datos, en el ahorro del dinero público, buscando acciones que ayuden a solventar problemas, y también en la salud (Joyanes, 2013). Además, también se pone al servicio de la ciudadanía acciones de mejora que favorecen al desarrollo y conexión con la localidad donde vive, llegando a desarrollar diferentes iniciativas.

El Big Data dispone del potencial de modificar el gobierno, incluso llegando a transformarlo de manera completa, y a la misma sociedad (López, 2013). Información nueva, noticias, hechos de interés, relaciones entre administraciones y otros indicadores que no están a la vista, ya que se encuentran inmersos en la gran cantidad de volumen, variedad y velocidad de datos existente.

\footnotetext{
${ }^{5}$ https://administracionelectronica.gob.es/pae_Home
} 
Dentro del sector público podemos destacar dos sectores que aprovechan y analizan de una manera profunda los datos masivos para poder conseguir una mejor gestión, si nos fijamos en los términos de eficiencia y eficacia. El Big Data está provocando un ahorro de diferentes costes en los diferentes sectores de la administración, además de un servicio mejorado a la ciudadanía.

\subsection{Salud}

En el mundo del ámbito sanitario disponemos de muchos caminos de los datos masivos. Si nos sumergimos en la actualidad, y nos fijamos en la crisis sanitaria global que está sucediendo, el SARS-CoV-2, o más conocido como coronavirus o Covid-19, podemos establecer algunos aspectos que se deberían tomar en cuenta por los responsables a la hora de llevar diferentes tratamientos de datos (Steta, 2020). Sobre todo, cuando nos referimos a datos pertenecientes a categorías especiales de datos como es el caso de los datos de salud.

El primer punto que se debe tener en cuenta es el tipo de datos que se están llegando a tratar. Hay casos en que los datos se procesan mediante herramientas de Big Data, son datos que no identifican a las personas, situación en la que no seria necesaria la aplicación de las disposiciones que ofrece el Reglamento General de Protección de Datos. Si los datos que se están solicitando se obtienen a través de mecanismos o herramientas tecnológicas, es altamente recomendable llevar a cabo evaluaciones de impacto para poder determinar lo especificado anteriormente, así como las medidas que, en su caso, se deban de implementar, dependiendo la situación en la que se encuentre cada caso.

$\mathrm{Si}$, por el contrario, se tratan datos que identifican o hacen identificable a una persona, es muy importante cumplir con ciertas medidas de forma previa al tratamiento, como una vez que se esté llevando el tratamiento de datos que anteriormente se ha mencionado. A continuación, aparecen los principales criterios que, se estima, deben ser considerados para dicho efecto (Steta, 2020: 38):

1. Evaluación de impacto.

2. Establecer las finalidades.

3. Determinar los datos que se van a recabar.

4. Establecer las medidas de seguridad aplicables a dichos datos.

5. Determinar los plazos de conservación de los datos.

6. Notificar en caso de cualquier violación de seguridad.

No debemos olvidar que, una vez tratados los datos de las personas usuarias del ámbito sanitario, muy importante, ya que las personas son lo más importante dentro de este ámbito, debemos ir al gráfico número 3.

Si nos fijamos en este gráfico, el Covid-19 es tratado de manera tardía, es decir, no se ve ni se llega a apreciar en el momento, sino tarde y cuando se dan sus consecuencias. Si hubie- 
ran aplicado el proceso de análisis de Big Data y la medicina preventiva previamente, podríamos haber obtenido diferentes resultados y haber podido atajar la pandemia de diferente manera.

Gráfico 3. Aplicación del Big Data en el sector de la salud

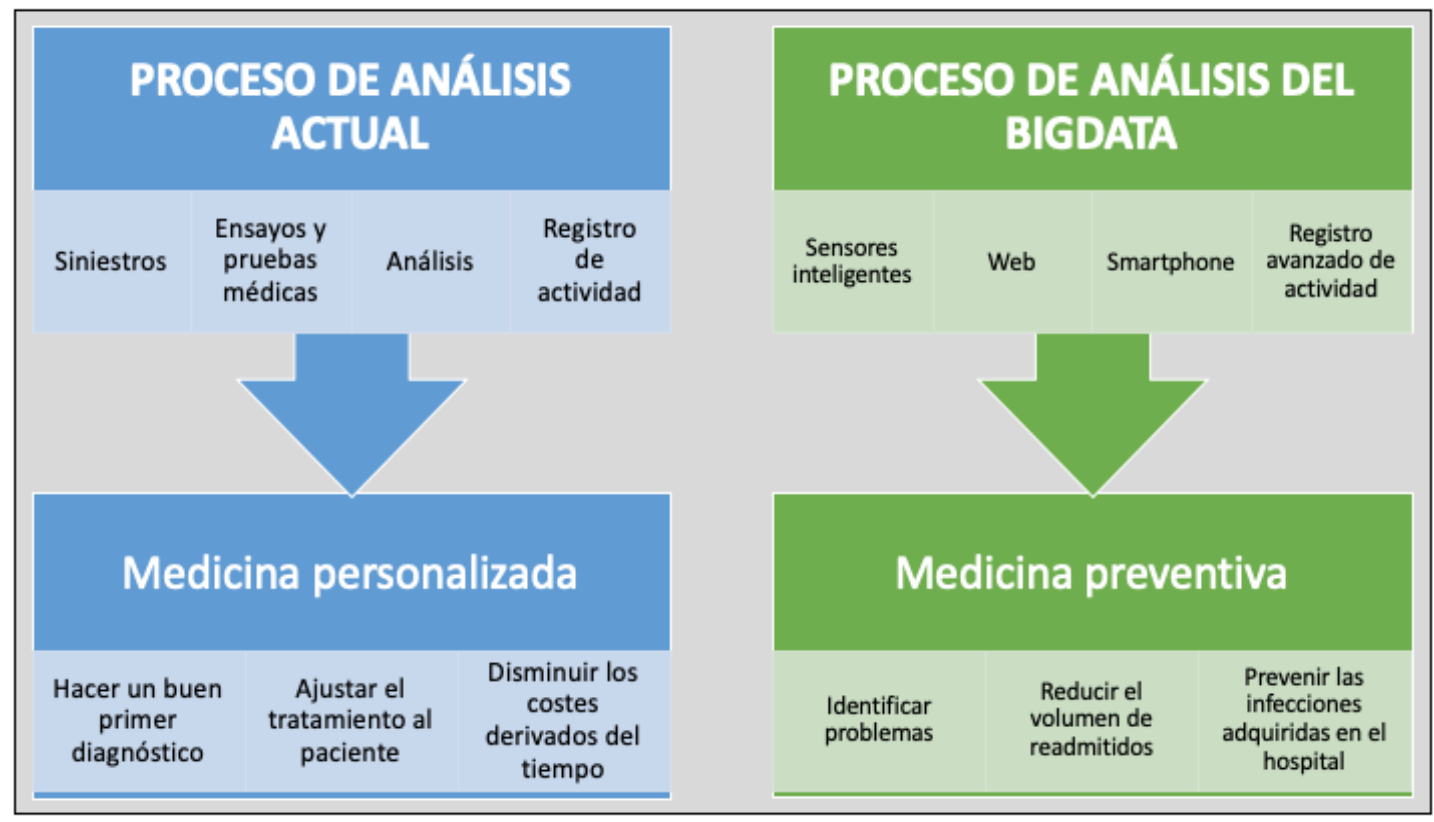

Fuente: elaboración propia, a partir de datos obtenidos de McKinsey (2013).

\subsection{Gestión urbana}

La gestión urbana es uno de los puntos más importantes para poder analizar el Big Data. Las ciudades generan una gran cantidad de información de distintos ámbitos, una cantidad de datos que ni un analista por sí solo podría llegar a analizar. Según la página web PowerData, cada día se producen más de 3.000 millones de datos, pero únicamente el 7\% de los datos se consideran que están estructurados de naturaleza. Para el autor inglés, Ajit Jaokar (2012), nos interesan destacar tres fuentes de información que son principales en relación al ámbito urbano: datos que son generados por sensores de distintas tipologías, datos de informes que son accesibles en plataformas de acceso abierto y las redes sociales.

En general, cada organismo ha experimentado una evolución diferente dependiendo de la cantidad de datos que procesa diariamente; pero esta evolución se ha producido bajo el modelo «silo» (Peñazola y Vargas-Perez, 2017). Este modelo nos indica que «las plataformas compatibles con múltiples tipos de datos y fuentes prosperarán, mientras que las diseñadas para ser utilizadas con una única fuente quedarán obsoletas» (Peñazola y Vargas-Pérez, 2017: 4). Además, es capaz de integrar y gestionar mucha más información dentro de sus sistemas, pero de una manera aislada respecto al resto de sistemas que conforman el organismo o, mejor dicho, la ciudad. En la actualidad, las ciudades que exprimen al 
Big Data: la revolución de los datos masivos en la Administración Pública | Lander Jauregi-Maza

máximo la potencialidad que les da el Big Data están trabajando para poder construir sistemas que consigan integrar o conectar todos los sistemas que registren datos de los que disponga dicha ciudad.

En este nuevo modelo de transformación de las ciudades, la ciudad estadounidense de Chicago es una de las que está más avanzada en la aplicación del Big Data. El avance que ostenta esta ciudad hace hincapié en la salud, en el transporte, en la educación, en la energía, etc. Este avance que se ha mencionado puede visualizarse en la imagen 1 del artículo. En esta imagen podemos ver cómo los datos de la ciudad de Chicago están en constante movimiento por toda ella, además de estar conectados con todas las bases operativas de análisis de datos.

Imagen 1. Mapa de conexión de datos en la ciudad de Chicago (EEUU)

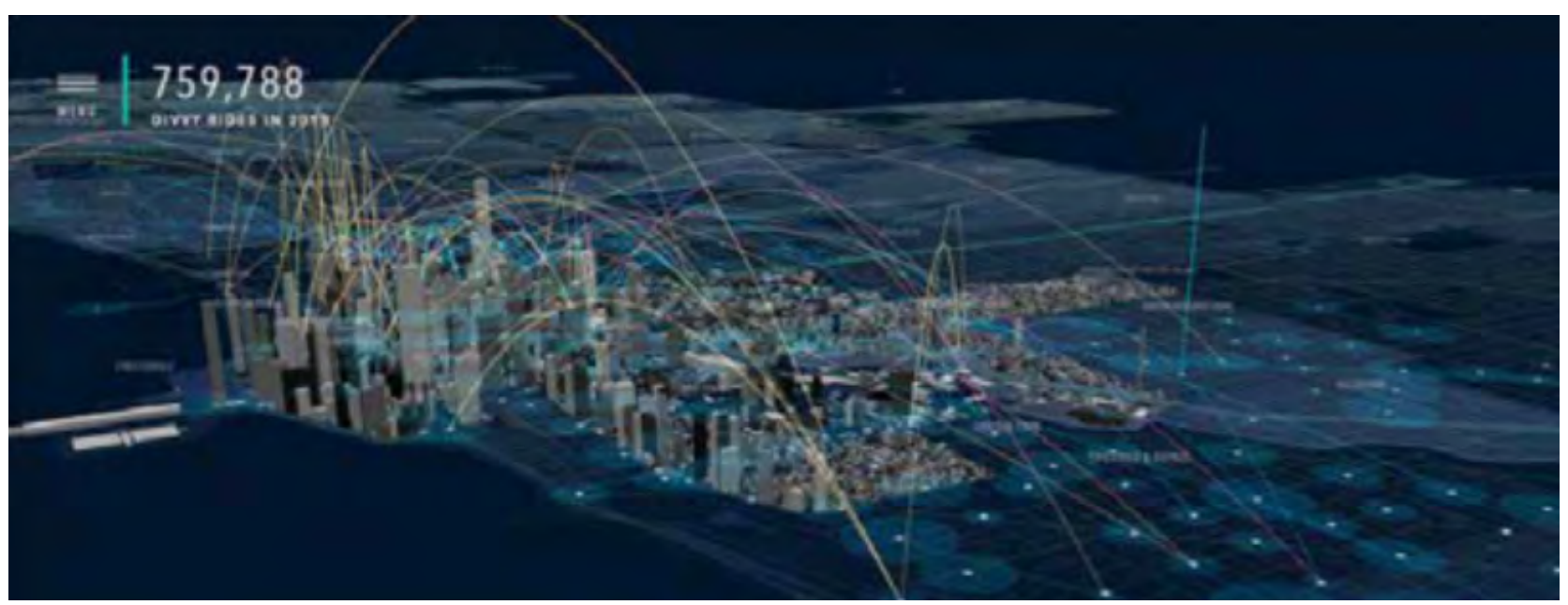

Fuente: imagen obtenida de architecture.org/exhibits/exhibit/chicago-city-of-big-data/

Podemos ver cómo Chicago está realizando un esfuerzo, al igual que el resto de las ciudades que están haciendo uso del Big Data. No solo para poder obtener gran cantidad de datos en tiempo real, sino para estudiar en cómo aportar esos datos en conocimiento útil y darles un buen uso. Por ejemplo, de cómo las ciudades explotan los datos para poder dar mayor valor añadido a su gestión y, además, una mejor atención a la ciudadanía (ver gráfico 4).

Chicago, asimismo, también permite la posibilidad de conectar a la juventud que está comprometida con esta causa a través de jornadas semanales llamadas «Noche de los Hackers». De este modo, los jóvenes que quieran colaborar y ayudar pueden hacerlo desarrollando técnicas de análisis de los algoritmos que ya existen, o incluso creando ellos mismos los suyos. Además, con estas jornadas se van a poder mejorar y perfeccionar las técnicas de análisis de los datos y solventar los fallos existentes. Sin ninguna duda, Chicago es una ciudad ejemplo de cómo la ciudad acerca los datos a la ciudadanía, y cómo la ciudadanía responde colaborando de una manera activa con la propuesta generando entre ambas partes una relación de «ambos ganamos» (Maroto, 2016). 
Gráfico 4. Ejemplos de explotación de datos con el Big Data en las ciudades

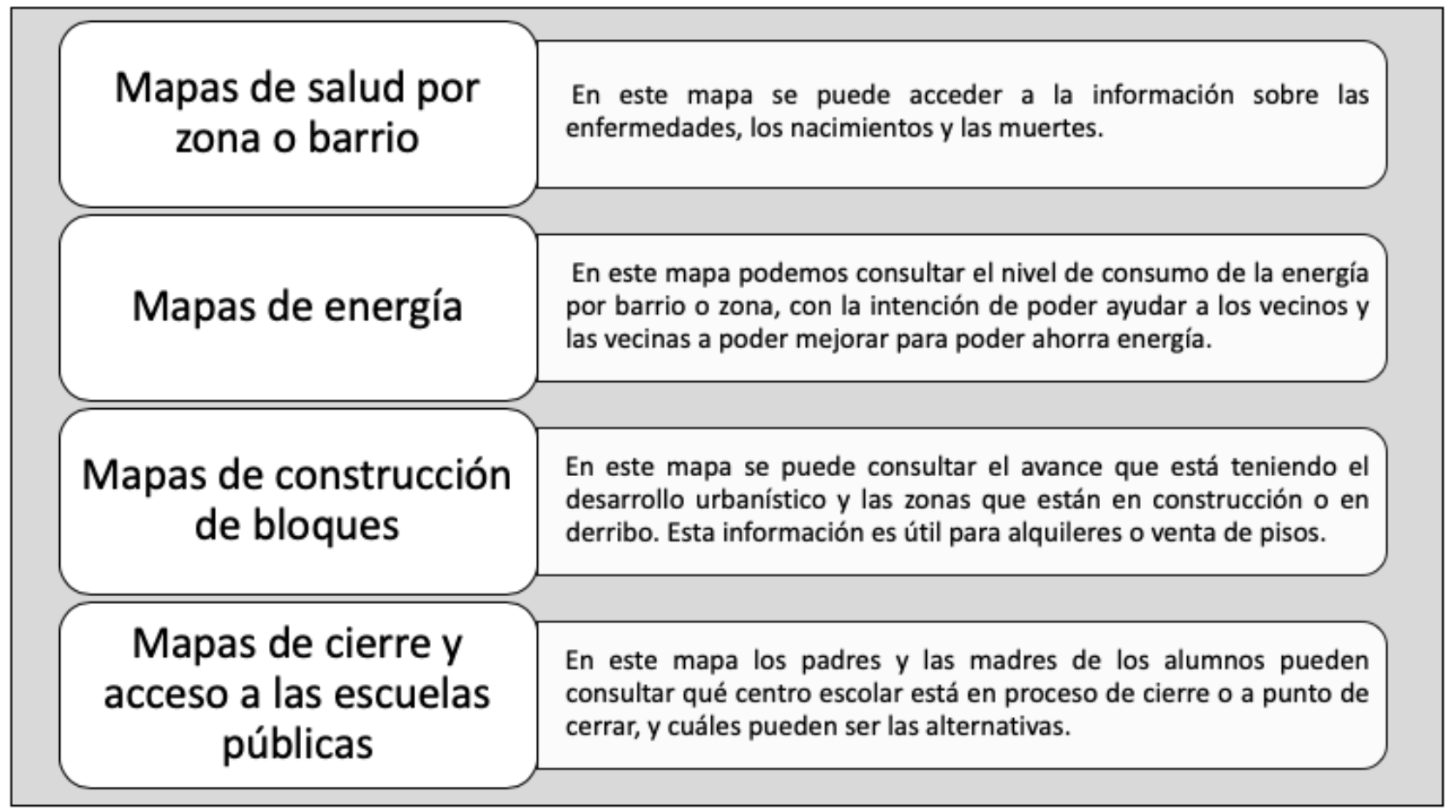

Fuente: elaboración propia, a partir de datos obtenidos de Jaokar (2012).

Las ciudades, sin embargo, también llevan a cabo otro tipo de tareas que, son utilizadas junto con el Big Data, como son:

\section{- Eficiencia y atención ciudadana.}

Hoy en día, se están haciendo cruces de datos municipales de contabilidad, asistencia de personas a eventos y equipamientos municipales con información obtenido mediante sensores que se instalan para poder medir la humedad, el tráfico, etc. Y con ellos podemos realizar un uso más eficiente de los sistemas de regado de los parques, la gestión de residuos y del transporte público. Además, también se están juntando los indicadores y sensores de las diferentes Fuerzas y Cuerpos de Seguridad para poder generar un mapa en el que podamos ver dónde se sitúan las alertas y avisos con diagnósticos producidos por algoritmos que incorporen datos de los diferentes sensores que están en la ciudad, así como la preparación de la población en núcleos muy poblados, frente a grandes contingencias, como pueden ser los terremotos o las grandes tormentas.

\section{- Seguridad.}

En el caso del gobierno estadounidense, podemos ver cómo ha creado un centro de excelencia en la ciudad de Nueva York que es capaz de poder gestionar miles y miles de fuentes de datos que se encuentren dispersas; así como habilitar la conexión de diferentes redes de datos (cámara de vigilancia, semáforos, sistemas de seguridad, etc.) de manera transparente para el operador que gestiona los datos en el Centro de Mando y Control, y aportar sensores virtuales que proporcionan novedosos tipos de información. Y todo ello se realiza en tiempo real. 


\section{- Gestión de eventos.}

Cuando se analizan los registros de transacciones de tarjetas de crédito que se realizan en los comercios de una ciudad, el Big Data proporciona una serie de recomendaciones objetivas para poder tener información sobre la celebración de un gran evento. Gracias a toda esta información que obtenemos sobre cómo se comporta la ciudadanía en relación a los comercios de las zonas, podemos identificar zonas o barrios que sean más activos, pero también las zonas que son más afectadas económicamente.

\section{- Tráfico.}

Las ciudades están en constante evolución sobre el crecimiento demográfico, y a una velocidad extrema, y para ello, el aumento del tráfico en las calles del municipio también es notorio. Esto nos lleva a que los coches sean una de las principales fuentes de contaminación municipal que llega a afectar, de manera directa, en la salud de la ciudadanía. El objetivo que se está buscando es la de un transporte urbano sostenible en el medio ambiente, que nos llegue a garantizar la competitividad y poder abordar las preocupaciones sociales. Todo esto nos lleva a un desafío de carácter común y urgente en las grandes ciudades europeas. En la tabla 1 podemos ver una pequeña síntesis de lo expuesto anteriormente.

Tabla 1. Principales ejes para el desarrollo de políticas públicas de movilidad sostenible a partir del Big Data

\begin{tabular}{|c|l|}
\hline Utilización de los datos & $\begin{array}{l}\text { La penetración de las TIC ha llegado a modificar las relaciones sociales, } \\
\text { además del comportamiento de los viajeros, y al mismo tiempo está pro- } \\
\text { porcionando una cantidad de datos que son heterogéneos, que son pro- } \\
\text { cedentes de sistemas de transportes inteligentes, redes sociales, etc. Las } \\
\text { distintas ciudades investigan cómo poder explotar la gran cantidad de } \\
\text { datos que se genera, y así crear patrones de movilidad. }\end{array}$ \\
\hline $\begin{array}{c}\text { Es muy interesante la investigación de las interacciones que se produ- } \\
\text { cen entre las redes sociales y el comportamiento de los viajeros. Esto nos } \\
\text { permite poder evaluar de una manera más completa las políticas públi- } \\
\text { cas de movilidad, en torno a la idea de un acceso compartido a todos los } \\
\text { recursos. Los nuevos modelos de comportamiento de viajeros son inte- } \\
\text { grados en las herramientas utilizadas para la simulación. }\end{array}$ \\
$\begin{array}{c}\text { Conexión entre } \\
\text { los diseñadores } \\
\text { del modelo, los tomadores } \\
\text { de decisiones } \\
\text { actores sociales }\end{array}$ & $\begin{array}{l}\text { El potencial que tienen incluido los sistemas de simulación urbana está } \\
\text { aún un poco explotado en los contextos de decisiones políticas. Existen } \\
\text { herramientas, como el análisis 3D virtuales, que nos permite la inte- } \\
\text { racción de los stakeholders con los resultados de la simulación y sirven } \\
\text { como una metodología para la evaluación conjunta. }\end{array}$ \\
\hline
\end{tabular}

Fuente: elaboración propia, a partir de datos obtenidos de «Evolutive User-centric Networks for Intraurban Accesibility».

\section{CONCLUSIONES}

Hoy en día el Big Data está presente en nuestras vidas. El uso de las TIC y las redes sociales han sido una de las grandes impulsoras para que podamos estar rodeados de datos. El Big Data ha tenido que crear un camino para poder llegar hasta nuestros tiempos, y de esa 
manera, han ido evolucionando las herramientas con las cuales se realizan la recogida de datos de Big Data.

En la evolución del Big Data hasta nuestros días, podemos ver una evolución de carácter más acelerada en los últimos tiempos. Si observamos el recorrido, vemos cómo las diferentes herramientas de recogida de datos han ido aumentando, hasta llegar a generar una gran cantidad de datos y, por tanto, de difícil manejo. La implantación del Big Data en todo los órdenes - y, por tanto, también en la administración pública - ha ido ayudando a las administraciones públicas a poder mejorar sus servicios, llegando a hacerlos más eficientes y eficaces. Las aplicaciones son múltiples: desde las gestiones más comunes, elaboración de predicciones de usos de servicios públicos como la sanidad a estimaciones de la contaminación de las ciudades, la regulación del tráfico, la reducción de la contaminación, etc., por poner solamente algunos ejemplos.

La relación del Big Data y el sector público es cada vez mayor. Las administraciones públicas hacen un uso cada vez más intensivo de los datos para poder elaborar nuevas políticas públicas en beneficio de la ciudadanía. Las Smart Cities ${ }^{6}$ fueron surgidas para poder lograr una mayor eficiencia y eficacia en la gestión de las ciudades, y de esta manera, poder dar un mayor fomento a la innovación y a la eficiencia en los servicios públicos locales. Es importante que en esta relación los datos sean utilizados para poder generar información y conocimiento, y de manera posterior, puedan ser aplicados a los diferentes sectores públicos (sanidad, seguridad, educación, etc.).

También podemos ver cómo el Big Data ha llegado a generar cambios significativos en el sector público. Junto con la llegada de las herramientas tecnológicas a nuestras manos tenemos la posibilidad de manejar una gran cantidad de datos. Las administraciones públicas gobiernan cantidades de datos muy voluminosas para generar información que pueda ser de utilidad a la ciudadanía, pero también para ellas mismas. Con la llegada del Big Data las gestiones de las administraciones públicas deberían ser mucho más rápidas, eficientes y eficaces. En consecuencia, desde la llegada del Big Data es lo que hemos visto, cambios significativos en el sector público.

Además, no podemos olvidar que cuanta mayor sea la extensión de la tecnología, mayores posibilidades de propagación podrán tener las herramientas del Big Data de manera global. Después de haber investigado sobre las herramientas que dispone el Big Data, se ha podido comprobar que la amplitud de la tecnología ha llegado a impulsar a esta gran cantidad de datos de una manera disparada. Las herramientas del Big Data se basan en todo tipo de herramientas informáticas, además de muchas otras. Los lenguajes informáticos de estas herramientas ayudan a generar los algoritmos que ayudan a extraer los datos, y

\footnotetext{
${ }^{6}$ Según la Wikipedia, una Smart City, o Ciudad Inteligente, es un «tipo de desarrollo urbano basado en la sostenibilidad que es capaz de responder adecuadamente a las necesidades básicas de instituciones, empresas, y de los propios habitantes, tanto en el plano económico, como en los aspectos operativos, sociales y ambientales». Disponible en: https://es.wikipedia.org/wiki/Ciudad_inteligente
} 
así, puedan llegar a leerse en las herramientas del Big Data que disponen todas las administraciones públicas. Estas herramientas se han ido expandiendo por todo el mundo, en mayor o menor medida. Esto ha ayudado a que los datos generados por una administración pública puedan ser leídos y extraídos (es decir, descargados y reutilizados) por otros sistemas en cualquier sitio del mundo.

Gracias a Ackoff, se ha podido comprobar que para poder generar un conocimiento necesitamos disponer de información. Para poder obtener información, sin embargo, necesitamos disponer de un bien preciado como son los datos. Sin los datos, como hemos visto en el apartado 3 del presente artículo, no vamos a poder fabricar ningún tipo de información, y mucho menos, conocimiento. Por eso se debe tener en cuenta que la recogida de datos es crucial; sin ellos, poco conocimiento e información vamos a poder disponer. Para ello, debemos tener presente la constante evolución de las diferentes herramientas del Big Data existentes, porque sin estas herramientas poca recogida de datos se podría realizar.

Por otra parte, si nos dirigimos a la aplicación del Big Data que se está dando en los diferentes ámbitos del sector público, hemos podido comprobar cómo el Big Data está dando resultados reveladores. Como se ha podido ver en el apartado 5 del presente artículo, la presencia del Big Data en la salud y en la gestión urbana tiene un peso determinante para adaptarse a los nuevos tiempos, porque con la gestión de los datos se puede llegar a conocer más a la ciudadanía y aportar información en un tiempo inmediato. El Big Data ha podido ayudar a realizar acciones que antiguamente podrían llegar a ser inasumibles y se pudieran realizar de una manera tan rápida, eficaz y eficiente o que, simplemente, se pudieran realizar. Han ayudado a las personas trabajadoras de esos espacios a poder dar una facilidad a la ciudadanía a la hora de poder realizar cualquier tipo de gestión o trámite. Sobre todo, en la gestión urbana se ha podido ver cómo el Big Data ha ayudado a poder disponer de datos en tiempo real y poder mostrarlos a la ciudadanía para que sepan en todo momento cómo está la situación de la gestión que quiera realizar (conocer la situación del tráfico, conocer cómo se encuentra la situación de los parques, saber cuándo llega el autobús a la parada, etc.). O incluso, para poder generar información con esos datos. Por ello, debemos tener en cuenta que el dato es la parte nutriente del Big Data, y no podemos dejarlo atrás.

Si hacemos referencia al sector de la gestión urbana, vemos la cantidad de datos que genera (tráfico, medio ambiente, transporte público, etc.). Una aplicación de todo esto la podemos observar en el fenómeno de las Smart Cities (Ciudades Inteligentes, en castellano). Las Smart Cities hacen uso de una gran cantidad de datos para poder responder de una manera eficaz y eficiente a los retos que tienen ante sí las grandes ciudades del siglo xxi, y con esos datos, poder tener información actualizada para las diferentes estrategias que se quieran realizar (medioambiental, movilidad, etc.). Por este motivo, la gestión de datos en el ámbito urbano es uno de los sectores más destacables del Big Data ${ }^{7}$, ya que sin la correcta gestión de los datos no se podrían llegar a desarrollar las Smart Cities.

\footnotetext{
7 En el territorio español, podemos destacar la elaboración del Libro Blanco de las Smart Cities en el año 2012 (http://www.innopro.es/pdfs/libro_blanco_smart_cities.pdf).
} 
Se ha podido observar que el Big Data no llega a dar los mismos resultados en todos los sectores. Se puede considerar mucho más adecuado en algunos concretos, por ejemplo, en el sector de la salud y en el sector de la gestión urbana. Pero, hay muchos otros sectores que sí hacen uso del Big Data, aunque sin llegar a obtener los beneficios que se esperaban. Estos sectores que no están tan beneficiados (hacienda, cultura, etc.), pueden llegar a utilizar el Big Data de una manera no tan usual como en los mencionados anteriormente, pero sí que les da una facilidad para poder extraer datos de diferentes temas que quieran poder analizar, y así, poder generar información.

En definitiva, es muy importante la gestión de los datos, ya que sin la correcta ejecución podemos estar apartando una cantidad de ellos que pueden ser muy útiles para poder generar información. Como hemos visto en los ejemplos expuestos en el anterior apartado, la correcta gestión de los datos ayuda a poder mejorar las labores administrativas diarias, y de esta manera, poder tener una mejor gestión para el beneficio de la ciudadanía. Para ello, se debe disponer de personal cualificado para poder gestionar esta gran cantidad de datos que está llenando las bases de datos de las administraciones públicas.

\section{BIBLIOGRAFÍA}

Ackoff, Russell (1989). «From Data to Wisdom». Journal of Applied System Analysis, 16, 3-9. Disponible en: http://www-public.imtbs-tsp.eu/ gibson/Teaching/Teaching-ReadingMaterial/Ackoff89.pdf

Bauzá, Felio José (2017). «Big data y open data en la administración turística: acceso y reutilización de información». R.V.A.P., 108, 19-41. Disponible en: https://doi. org/10.47623/ivap-rvap.108.2017.01

Beltrán-Orenes, Pilar y Martínez-Pastor, Esther (2016). «Grado de cumplimiento de las leyes de transparencia, acceso y buen gobierno y de reutilización de los datos de contratación de la Administración Central del Estado». El profesional de la información, N. 4, 557-567. Disponible en: https://doi.org/10.3145/epi.2016.jul.05

Groves, Peter et al. (2013). «The "bigdata” revolution in healthcare. Acceleration value and innovation». Center for US Health System Reform Business Technology Office, N. 1, 1-19. Disponible en: https://www.ghdonline.org/uploads/Big_Data_Revolution_in_ health_care_2013_McKinsey_Report.pdf

Joyanes, Luis (2013). Big Data. Análisis de grandes volúmenes de datos en organizaciones. México: Alfaomega.

López, David (2013). Análisis de las posibilidades de uso de Big Data en las organizaciones. Trabajo de Fin de Máster. Santander: Universidad de Cantabria. 
Big Data: la revolución de los datos masivos en la Administración Pública | Lander Jauregi-Maza

Maroto, Chema (2016). "Big Data y su impacto en el sector público». Business Review, N. 256, 16-25. Disponible en: https://www.harvard-deusto.com/big-data-y-su-impacto-en-el-sector-publico

Mayer-Schönberger, Viktor y Cukier, Kenneth (2013). Big Data. La revolución de los datos masivos. Madrid: Turner Noema.

Mirón, Felipe et al. (2017). "Análisis de la estrategia Big Data en España». Planeta, N. 1, 1-23. Disponible en: https://packnet.es/wp-content/uploads/2020/03/Ana\%CC\%81lisisestrategico-Big-Data-en-Espan\%CC\%83a_may18.pdf

OECD (2013). «Exploring Data-Driven Innovation as a New Source of Growth: Mapping the Policy Issues Raised by «Big Data»». OECD Digital Economy Papers, N. 222, OECD Publishing, Paris.

OSPI (2016). «Big data en las administraciones públicas» en Observatorio Sector Público IECISA, disponible en: https://www.ospi.es/es/informes/detalle/Big-Data-en-las-Administraciones-Publicas/

Peñazola, Juan Luis y Vargas-Pérez, Carmen (2017). «Ecosistema de Big-Data: Tendencias y Retos en el 2018». Spain Business School, 1-7. Disponible en: https://www.researchgate.net/publication/321906722_Ecosistema_de_Big-Data_Tendencias_y_Retos_en_ el_2018

Steta, Gerardo Antonio (2020). Big Data y Protección de Datos en Tiempos del Covid-19. Trabajo de Fin de Máster. Madrid: Universidad Europea. 